\title{
BIG BUSINESS: O EMPREGO DO BIG DATA NA MELHORIA DA GESTÃO DE NEGÓCIOS
}

\section{ARTIGO ORIGINAL}

COSTA, Caroline Pereira Do Nascimento $\mathrm{Da}^{1}$

COSTA, Renato Dos Santos Da ${ }^{2}$

CONRADO, Luciane Medeiros De Souza ${ }^{3}$

COSTA, Caroline Pereira Do Nascimento Da. COSTA, Renato Dos Santos Da. CONRADO, Luciane Medeiros De Souza. Big Business: 0 emprego do Big Data na melhoria da gestão de negócios. Revista Científica Multidisciplinar Núcleo do Conhecimento. Ano 05, Ed. 03, Vol. 06, pp. 29-40. Março de 2020. ISSN: 2448-0959, Link de acesso: https://www.nucleodoconhecimento.com.br/administracao/melhoriada-gestao-de-negocios

${ }^{1}$ MBA em Gestão de Processos - FGV/RJ. MBA em Gestão de Projetos - Unopar. MBA em Gestão de Negócios com ênfase em Auditoria e Controladoria - Ucam/RJ. Licenciatura em Administração - Ucam/RJ. Bacharelado em Administração UniSuam/RJ.

${ }^{2}$ Mestrando em Novas Tecnologias Digitais na Educação/UniCarioca RJ. Mestre em Educação/Universidad de Jaén (Espanha). MBA em Gestão de Projetos/FGV RJ. Pós Graduado em Tecnologia da Informação e Comunicação/Ucam RJ. Pós Graduado em Docência do Ensino Superior/Ucam RJ. Licenciado em Matemática/UniFranca SP. Licenciado em Informática/Ucam RJ. Tecnólogo em Processamento de Dados/UniverCidade RJ. Professor do Instituto Federal de Ciência e Tecnologia do Rio de Janeiro - IFRJ.

${ }^{3}$ Doutorado em Doutorado em Letras Universidade Federal Fluminense. Mestrado em Ciências da Arte. Graduação em Ciências Sociais. 


\section{RESUMO}

Este artigo tem como objetivo principal apresentar e refletir o emprego do Big Data e do Business Intelligence (BI), os vislumbrando, em suas diferentes concepções e aplicações, como instrumentos potencializadores de melhorias na gestão de negócios. Para sobreviver a "quarta onda industrial" as organizações precisam cada vez mais de inovações e otimizações em seus processos de gestão e contato com seus públicos-alvo. A predição de dados do sistema Big Data traz a luz e a inferência tanto nos conhecimentos das necessidades do mercado, quanto nos métodos de análise de eficácia nos procedimentos e sistemas avaliativos das organizações inclusas nas sociedades do conhecimento e da informação. O trabalho se justifica na importância para o desenvolvimento das Ciências, Organizações e Negócios em conhecer a utilização das tecnologias para explorar formas eficazes nos processamentos de informações, tomadas de decisões e conexões novas e eficazes na direção das demandas, especialmente nas relações com os clientes em um âmbito de negócios. Utiliza-se a pesquisa bibliográfica e documental para revisar a literatura da área, democratizando o conhecimento sobre Big Data e o diferenciando do BI. Conclui-se que a tecnologia do Big Data, usada no âmbito da gestão, pode permitir que as organizações busquem o que chamam de "oceano azul", ou seja, uma distância de seus concorrentes, promovida pelas melhores percepções do mercado oriundas do processamento de informações e da maior aproximação com os clientes.

Palavras-chave: Big Data, sociedade da informação, tecnologia da informação, Business Intelligence, gestão de processos.

\section{INTRODUÇÃO}

Em uma "Sociedade da Informação" onde os bens e equipamentos não são mais o grande ativo das instituições, o conhecimento, passa a ser o recurso mais valioso e importante das organizações. Observamos que o armazenamento de dados históricos para consulta através de bancos de dados não é mais suficiente para que as 
instituições do presente possam abarcar na "próxima onda" seja ela agrícola, industrial, informacional, dentre outras (CHIAVENATO, 2006).

Obter uma informação antecipadamente pode representar a diferença entre o fracasso e o sucesso, em qualquer âmbito, e para isso há quem recorra a diferentes fontes e utilize de inúmeros recursos. De qualquer modo, o acaso persegue a previsão e daí entra a probabilidade e a inferência, ramos da estatística que são úteis para analisar situações de maneira consciente.

Mas hoje, diante da complexidade e volume dos dados, as organizações não seriam capazes de utilizar a estatística a seu favor sem o uso dos computadores e de toda uma tecnologia que compõe a Ciência de Dados, capaz de definir processos, modelos, e tecnologias que estudam os dados durante todo o seu ciclo de vida: da produção ao descarte, (AMARAL, 2016).

Então, utilizando o computador como ferramenta e fazendo uso do Big Data para predizer comportamentos e tendências para é possível otimizar investimentos, tornando esse uso cada vez mais importante para as grandes tomadas de decisão que podem implicar mudanças organizacionais abruptas para adaptação a um mercado cada vez mais volátil. Em contrapartida, já estamos vivendo o fechamento e o encerramento de empresas que não se prepararam para estes ambientes de múltiplas informações, informações internas de apoio à decisão, inteligência de negócios (B.I.) e informações da sua concorrência (MACHADO, 2018).

A quantidade expressiva de dados que estão sendo produzidos diariamente graças à Internet e aos recursos atuais de informática, não seria sequer imaginável em uma era anterior. Um conhecimento valioso às organizações se encontra encoberto pelo volume de informações desconectadas.

Esse artigo demonstra de acordo com a literatura existente, as vantagens do Big Data em consonância do Business Inteligence nas decisões das empresas. 
Além dessa introdução, na seção 2 apresenta-se literatura sobre Big Data, enquanto na terceira seção observa-se a literatura de Business Inteligence (BI). Na seção 4 compara-se as vantagens e semelhanças do Big Data e BI. E por fim, na última seção encontra-se a conclusão.

\section{BIG DATA}

As organizações precisam cada vez mais analisar os mais diversos tipos de dados gerados pelos seus clientes para melhor conhecê-los e assim poder cada vez mais oferecer produtos e serviços aliados às suas necessidades. Esses dados estes são gerados a todo momento seja, por exemplo, quando realizamos um consumo através do cartão de crédito, quando escrevemos um post em uma rede social ou até mesmo ao se deslocar de posse do celular online e com gps habilitado. "Big Data é uma tecnologia que permite o processamento de informações com alto desempenho e disponibilidade. Sistemas relacionados à Big Data têm adquirido cada vez mais importância nos negócios nos últimos anos" (CHEN; CHIANG; STOREY, 2012).

O conceito de Big Data diz respeito justamente a essa crescente produção de dados, "seja por aplicações corporativas, seja pela Internet e suas redes sociais, assim como smartphones e celulares, equipamentos leitores de RFID e câmeras de controle de vídeo para segurança e tráfego etc.," (MACHADO, 2018).

O Big Data é um fenômeno, não é algo estruturado como um banco de dados que teve uma tabela projetada e foi alimentado sistemicamente por uma instituição, o Big Data surgiu graças ao aumento exponencial do uso de aplicações e serviços na Internet, aliado a redução no custo de armazenamento que de certa forma extingue a necessidade de descarte de dados quaisquer, que posteriormente percebendo se 0 valor que estes dados possam vir a representar caso venham a servir de insumo para ferramentas de extração de informações usadas na Ciência de Dados.

Nesse sentido, as empresas atuais devem ter consciência do aumento do volume, velocidade e variedade de dados, para fins de tomada de decisão em seus negócios. 
Os grandes dados são uma consequência do ambiente digital de hoje, que gera dados que fluem continuamente de todas as direções a uma velocidade e volume sem precedentes, e esses dados geralmente requerem limpeza. Eles são considerados "sujos" principalmente por causa da fusão de múltiplas fontes (RATNER, 2011).

Quando o termo sujo é empregado, compreende-se que os dados do Big Data não possuem significado, como a mais básica definição que o termo "dado" representa, precisando ser analisados com poderosas ferramentas computacionais de extração e transformação das informações, como poderosas aplicações de ETL (Extract Transform Load).

Estudos comprovam que empresas que investem em tecnologias como Big Data estão vivenciando crescimentos $50 \%$ superiores a empresas que não investem em novas tecnologias (DELL, 2015).

Figura 1: Médias de taxas de crescimento frente a nova tecnologia

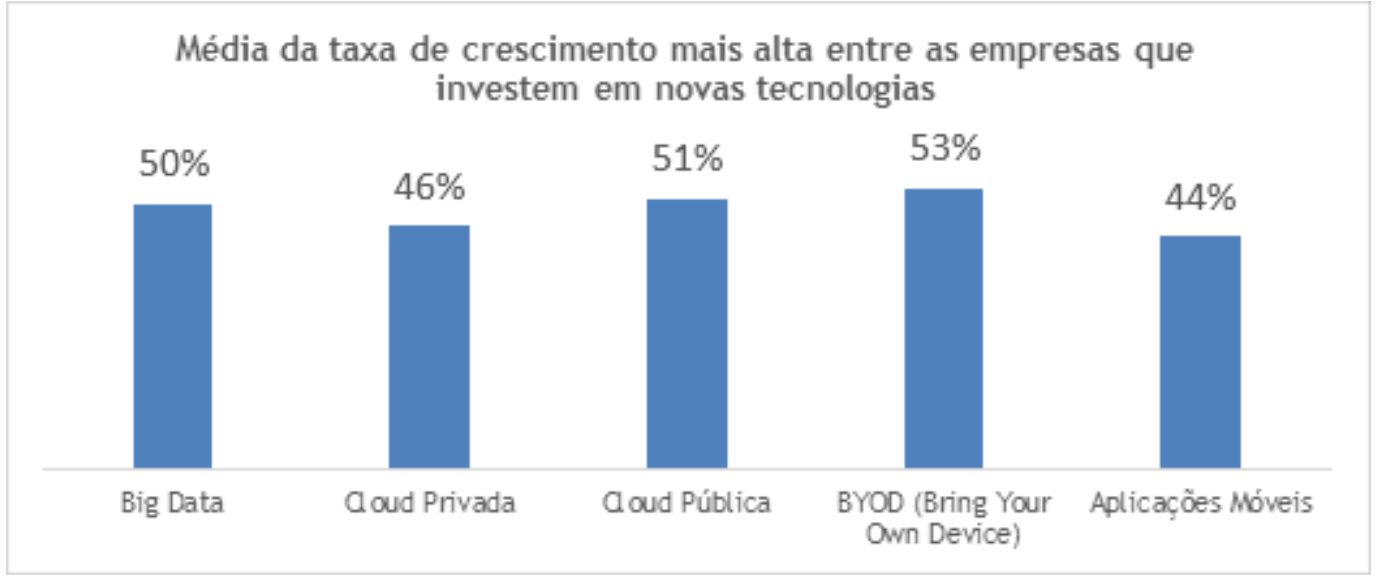

Fonte: https://www.camara-e.net/2015/10/20/empresas-que-investem-em-cloudcomputing-mobilidade-e-big-data-tem-crescido-ate-53-mais-rapido Acessado em 03/03/2020

"As empresas por sua vez que não souberem usar Big Data vão desaparecer, engolidas por concorrentes com menos custos, mais qualidade e clientes mais satisfeitos" (AMARAL, 2016).

Disponível em: https://www.nucleodoconhecimento.com.br/administracao/melhoria-da-gestao-denegocios 
As técnicas de Big Data podem proporcionar melhores resultados a uma organização. Através da obtenção e análise de informações sobre múltiplos consumidores é possível potencializar a oferta de produtos e serviços de modo cada vez mais "individualizado" para que o consumidor receba contato ofertando algo que realmente ele demanda, tais ações irão resultar em um maior engajamento com a marca.

Big Data Management (BDM) é um conjunto de ações para promover a coleta, armazenagem, organização, administração, governança e entrega de grandes volumes de dados, tendo em vista assegurar um alto nível de qualidade e torná-los acessíveis para as aplicações de Business Intelligence e Big Data Analytics. O Big Data Management migra, integra e prepara os dados para serem utilizados na geração de relatórios, painéis de indicadores de desempenho (dashboards) e na formação da inteligência de negócios, especialmente voltada para a preditividade de novas tendências, assim como gerencia o ciclo de vida da informação da sua origem até o seu descarte (TIEN, 2013, pag. 127).

\subsection{BIG DATA - TEORIA DOS Vs}

O Big Data pode ser definido como sendo um ativo de alto volume, velocidade e variedade de informação que exige custo-benefício, de formas inovadoras de processamento das informações para maior visibilidade e tomada de decisão.

Para alguns autores o Big Data apresenta três características básicas definidoras: volume (taxa de crescimento e taxa de dados), variedade (tipos de dados, como logs de sensores, microblogs - como Twitter e Facebook) e velocidade (a velocidade da fonte de fluxo de dados). Esses três termos são muito utilizados quando se trata de discussões Big Data e muitas vezes eles são chamados de "os 3 Vs" (ZIKOPOULOS; EATON, 2011).

Já para Taurion (2012), o conceito completo do Big Data está elencado em cinco princípios e cada um deles impacta diretamente no desempenho das soluções RC: 46711

Disponível em: https://www.nucleodoconhecimento.com.br/administracao/melhoria-da-gestao-denegocios 
tecnológicas disponibilizadas no mercado. Esses princípios são descritos como os " 5 V's" do Big Data: volume, velocidade, variedade, veracidade e valor.

Taurion (2012) ratifica ainda que o volume é a dimensão mais comuns no conceito de Big Data visto que a sociedade produz volumes de dados escaláveis a cada dia.

Em termos de quantidade de dados, é difícil acreditar que um dispositivo de memória de massa como um disco rígido já foi chamado de winchester, em um passado não muito distante, devido a uma analogia onde seus 22 megabytes correspondiam ao popular calibre de um rifle muito usado nos EUA no século XIX. Hoje em dia petabytes ainda estão muito distantes do uso doméstico, mas já representam uma realidade frente ao Big Data, em um cenário onde o baixo custo de armazenamento permanente favorece cada vez mais a manutenção dos dados, ainda que não se saiba exatamente se haverá ou não serventia no presente momento o descarte dos dados tem se tornado cada vez mais incomum.

Por isso, Big Data tem atraído atenção pela acelerada escala em que volumes de dados cada vez maiores são criados pela sociedade (velocidade). Assim, terabytes, petapytes e eventualmente exabytes de dados são gerados por seres humanos e por máquinas tendo como desafio a forma de armazenar, proteger e tornar acessíveis tais dados (PREIMESBERGER, 2011).

Quanto à variedade, comenta Taurion (2012), que dados estruturados, semiestruturados e não estruturados podem ser citados. Os dados estruturados são bem definidos e possuem um formato previsível e periódico. Eles são manipulados por um sistema de gerenciamento de banco de dados (SGBD) e consiste em registros, atributos, chaves, índices, planilhas ou tabelas. Como regra, a maioria das unidades de dados no ambiente estruturado pode ser localizada de forma muito rápida e fácil.

De acordo com Taurion (2012) os dados não estruturados são a imensa maioria produzida na Internet. Exemplos de dados não estruturados são vídeos digitais, imagens, emoticons, twetts, posts em redes sociais, likes no Youtube, stories do Instagram, arquivos de registros (logs), dentre outros.

Disponível em: https://www.nucleodoconhecimento.com.br/administracao/melhoria-da-gestao-denegocios 
Em regra geral, os dados não estruturados são bastante desajeitados para o acesso, onde as sequências de dados longas devem ser pesquisadas sequencialmente para encontrar uma determinada unidade/padrão de dados. Existem muitas formas e variações de dados não estruturados. Talvez a forma mais comum de dados não estruturados seja o texto (RATNER, 2011).

Quanto ao valor, o Big Data só faz sentido se a análise dos dados compensar o custobenefício de sua coleta, armazenamento e processamento. Para que um dado tenha qualidade deve ser exato, íntegro, consistente e relevante (WEBER, 2009).

O Big Data transforma a maneira como entendemos e exploramos o mundo. Na era dos pequenos dados, éramos motivados por hipóteses sobre como o mundo funcionava, as quais depois tentávamos validar coletando e analisando dados. No futuro, nossa compreensão será motivada mais pela profusão de dados do que por hipóteses. Essas hipóteses geralmente surgem de teorias das ciências naturais ou sociais, que por sua vez ajudam a explicar e/ou prever o mundo. À medida que passamos de um mundo movido por hipóteses para um mundo movido por dados, talvez nos sintamos tentados a pensar que já não precisamos de teorias. (SCHÖNBERGER; CUKIER, 2013, pag. 48).

É necessário, igualmente, que os dados façam sentido e que sejam autênticos. A veracidade é a qualidade do que é verdadeiro e exato, sendo assim, informações verdadeiras têm relação com informações exatas, íntegras, consistentes e relevantes podendo ser utilizadas pelos gestores para responder aos desafios estratégicos e operacionais nas empresas.

\section{O BUSINESS INTELLIGENCE (B.I.)}

Muitos confundem o conceito de Business Intelligence(B.I. ou Inteligência de Negócios) com o de Big Data, embora as diferenças sejam sutis, os processos têm características particulares e complementares. Entender o que cada um representa é fundamental para garantir que as empresas consigam utilizá-los a seu favor.

Disponível em: https://www.nucleodoconhecimento.com.br/administracao/melhoria-da-gestao-de- 
Sendo assim, podemos definir o B.I. como a inteligência de negócios ou empresariais que empregam da melhoria ou da prospecção de novos insumos, revelando dados de caráter informacional e estratégico.

Muitos gestores usam o B.I. como resposta para definir as novas estratégias e iluminar os novos direcionadores quando de uma reformulação organizacional.

"O processo de tomar decisão começa com uma situação de frustração, interesse, desafio, curiosidade ou irritação. Há um objetivo a ser atingido e apresenta-se como um obstáculo, ou acontece uma condição que se deve corrigir, ou está ocorrendo um fato que exige algum tipo de ação, ou apresenta-se uma oportunidade que pode ser aproveitado" (MAXIMIANO, 2009, p. 58)

A função primordial do Business Intelligence é gerar informações relevantes para a tomada de decisões a partir do processamento de dados aparentemente desconexos, guiando gestores com um embasamento robusto, através de um painel repleto de gráficos e informações (dashboard) necessárias para orientar os gestores, no lugar de uma imensidão de dados oriundos de gigantescos relatórios inúteis, permitindo assim, vantagem competitiva frente ao mercado.

Para redefinir estes dados "brutos" do conhecimento a partir de transações do dia a dia são utilizadas ferramentas como Customer Relationship Management (CRM) para gestão de relacionamento com o cliente, Data Mining (DM) para mineração de dados, Data Warehouse ou armazém de dados, Enterprise Resource Planning (ERP) gerindo recursos empresariais através de seus diversos módulso, dentre outras tantas ferramentas de gestão da informação.

O Business Intelligence é o resultado do processamento de dados que serão coletados, organizados e transformados em informações, analisadas e contextualizadas para transformarem-se em inteligência que, por sua vez, possibilitará a geração de vantagem competitiva para a organização e ajudam os usuários a 
tomarem melhores decisões estratégicas (DUMONT; RIBEIRO; RODRIGUES, 2006; TURBAN; RAINER; POTTER, 2005).

O Business Intelligence (B.I.) não só apoia o processo de tomada de decisão, como também permite que as organizações tenham melhores percepções em relação às suas operações através da aplicação das técnicas de análise de dados às suas informações, além de contribuir para a gestão da qualidade, detectando erros nos processos da empresa e avaliação desempenho da produtividade dos funcionários por meio de indicadores de desempenho.

Os projetos de B.I. costumam gozar de certa autonomia organizacional diante do fato de se relacionar diretamente com as mais altas áreas estratégicas da instituição, seus resultados são insumos indispensáveis tanto para o planejamento a médio e longo prazo quanto para ações que venham a necessitar ser tomadas de forma rápida, precisa e ao mesmo tempo eficaz e consequente.

\section{BUSINESS INTELLIGENCE X BIG DATA}

A principal diferença entre o B.I. e o Big Data é que este busca o "o quê" e não "o porquê" dos fatos, ou seja, enquanto o B.I. consiste em olhar o passado dos dados e extrair relatórios ou dashboards para apresentações executivas, o Big Data oferece um universo de novas possibilidades e perguntas sobre fatos que ainda não conhecemos, aplicando identificação de padrões, relacionamento entre variáveis e dados, analises estatísticas quantitativas e qualitativas com caráter preditivo.

A semelhança entre o B.I. e o conceito de Big Data nos faz cada vez mais rumar para o conceito de Big Business, onde "o B.l. é o sustentáculo do Big Data" (MACHADO, 2018).

Cabe salientar que a estratégia de adoção do Big Data está conectada a estratégia organizacional, que deverá além de colaborar com a decisão quanto a quais recursos analíticos serão necessários deverá também propor como e onde eles poderão ser aplicados (NOVO; NEVES, 2013). 
Indubitavelmente as empresas que vinham investindo em B.I. tendem a naturalmente embarcar em projetos de Big Data, estes elementos devem coexistir, sendo que o B.I. é mais dependente dos dados estruturados existentes em tabelas de bancos de dados da própria organização, enquanto o Big Data soma dados não estruturados heterogêneos e das mais variadas origens externas, além de implicar a utilização de estatísticos para fornecer as análises de negócios validas a uma organização.

\section{CONCLUSÃO}

Esse artigo evidenciou a importância do Big Data e do Business Intelligence nas decisões das empresas.

Concluiu-se que as ferramentas digitais eram primordiais para que gestores pudessem compreender com mais facilidade tendências e padrões, para que assim organizassem suas estratégias de negócios e através das alterações das variáveis conhecidas, acompanhassem em seus dashboards os futuros cenários, abarcando modelos com as possíveis previsões diante de cada escolha, e assim pudessem previr melhor os riscos e oportunidades otimizando as suas escolhas.

Observou-se que um conhecimento valioso demais às organizações encontrava-se encoberto pelo volume de informações sem significado e o Big Data era a tecnologia que ao ser adotada permitiria atribuir o valor necessário desempenhando o processamento de dados com alto desempenho e disponibilidade e extraindo as mais diversas informações sob demanda de acordo com as estratégias de cada segmento de negócio. Sistemas relacionados à Big Data adquiriram cada vez mais espaço importância nos últimos anos e o objetivo era predizer informações, melhorando a eficácia na tomada de decisões e mantendo as organizações que os exploram a frente de seus concorrentes em um mercado cada vez maior e ao mesmo tempo cada vez mais compartilhado e concorrido, a Internet. 


\section{REFERÊNCIAS}

AMARAL, Fernando. Introdução a Ciência de Dados: Mineração de Dados e Big Data. Rio de Janeiro: Altabooks, 2016. 320p

BEUREN, I. M. Gerenciamento da informação: um recurso estratégico no processo de gestão empresarial. São Paulo: Atlas, 2000. 104p

CARVALHO, Izabel Cristina Louzada. KANISKI, Ana Lúcia. (2000) A Sociedade do Conhecimento e o Acesso a Informação: Para que e Para Quem? Revista Ciência da Informação Volume 29 Número 3.

CHEN, H.; CHIANG, R. H. L.; STOREY, V. C. Business Intelligence and Analytics: From Big Data to Big impact. Mis Quarterly, v. 36, p. 1-22, December 2012. ISSN.

CHIAVENATO, Idalberto. Princípios da Administração: o essencial em teoria geral da administração. Rio de Janeiro: Elsevier, 2006. 458p

CUKIER, Kenneth; SCHÖNBERGER, Viktor Mayer. Big Data: como extrair volume, variedade, velocidade e valor da avalanche de informação cotidiana. $1^{\underline{a}}$ ed. Editora Campus, 2013.

DAVENPORT, T., Barth, P., \& Bean, R. (2012). How 'Big Data' is Different. MIT Sloan, 2012.

DELL COMPANY INFORMATION. Global Technology Adoption Index 2015. USA. Disponível em https://www.infopointsecurity.de/medien/gtai_2015_results_deck_final_for_web_101115.pdf acessado em 03/03/2020.

FELIX, Bruno Muniz. TAVARES, Elaine. CAVALCANTI, Ney Wagner Freitas. (2018) Fatores Críticos de Sucesso Para Adoção de Big Data no Varejo Virtual: Estudo de Caso do Magazine Luiza. Revista Brasileira de Gestão de Negócios. 
GONÇALVES, M. M. S. Gestão de Recursos Humanos Como um Diferencial Competitivo. Belo Horizonte: ECX Card, 2012

LAUDON, Kenneth C.; LAUDON, Jane Price. Sistemas de informação. 4. ed. LTC: Rio de Janeiro, 1999

MACHADO, F. N. R. Tecnologia e Projeto de Data Warehouse. 2ª edição. São Paulo: Érica, 2004.

MACHADO, Felipe Nery Rodrigues. Big Data: O futuro dos dados e aplicações. São Paulo: Érica, 2018. 224p

MAXIMIANO, Antonio Cesar Amaru. Introdução à Administração. Ed. Compacta. São Paulo: Atlas, 2009.

NOVO, R.; NEVES, J. M. S. D. Inovação na inteligência analítica por meio do Big Data: característica de diferenciação da abordagem tradicional. VIII Workshop de Pósgraduação e Pesquisa do Centro Paula Souza. São Paulo: [s.n.]. 2013. p. 32-44.

ONG, I.; Siew, P.; Wong, S. A Five-Layered Business Intelligence Architecture. IBIMA Publishing, v. 2011, 2011.

PREIMESBERGER, C.. Big ideas about Big Data. eWeek, 15 aug. 2011.

RATNER, Bruce (2011). Statistical and Machine-Learning Data Mining Techniques for Better Predictive Modeling and Analysis of Big Data. Second Edition.

TAURION, C. (2012). Você realmente sabe o que é Big Data? IBM DeveloperWorks, Apr, 30.

THOMPSON, W. J. J.; VAN DER WALT, J. S., 2010, "Business Intelligence in the cloud". South Africa Journal of Information Management, v. 12, n. 1 (Dec), pp. 445450. 
TIEN, J. M. (2013). Big Data: Unleashing information. Journal of Systems Science and Systems Engineering, 22(2), 127-151.

TURBAN, Efraim. et al. . Business Intelligence: um enfoque gerencial para a inteligência do negócio. Porto Alegre: Bookman, 2009. 253p.

TURBAN, Efraim; RAINER, R. Kelly; POTTER, Richard E. Administração de tecnologia da informação: teoria \& prática. Rio de Janeiro: Elsevier, 2005.

VIANNA, W. B.; DUTRA, M. L.; FRAZZON, E. M. Big data e gestão da informação: modelagem do contexto decisional apoiado pela sistemografia. Informação \& Informação, v. 21, n. 1, p. 185-212, 2016. DOI: 10.5433/1981-8920.2016v21n1p185 Acesso em: 03 mar. 2020.

WEBER, C. A. A data envelopment analysis approach to measuring vendor performance. Supply Chain Management, v. 1, n. 1, p. 28-39, 2009.

ZIKOPOULOS, Paul e EATON, Chris (2011). Understanding Big Data Analytics for Enterprise Class Hadoop and Streaming Data.

Enviado: Fevereiro, 2020.

Aprovado: Março, 2020. 